\title{
Dairy products and prevention of type 2 diabetes: implications for research and practice
}

\author{
Maria Kalergis $^{1 *}$, Sylvie S. L. Leung Yinko ${ }^{2}$ and Roxana Nedelcu ${ }^{3}$ \\ 1 Dairy Farmers of Canada, Montreal, QC, Canada \\ 2 Division of Clinical Epidemiology, McGill University Health Centre, Montreal, QC, Canada \\ ${ }^{3}$ School of Dietetics and Human Nutrition, McGill University, Montreal, OC, Canada
}

Edited by:

Catherine Chan, University of Alberta, Canada

\section{Reviewed by:}

Melissa Irene March, Beth Israel Deaconess Medical Center, USA Rhonda C. Bell, University of Alberta Canada

Spencer Proctor, University of

Alberta, Canada

*Correspondence:

Maria Kalergis, Dairy Farmers of Canada, 1801 McGill College Avenue \#700, Montreal, OC H3A 2N4, Canada e-mail:maria.kalergis@dfc-plc.ca
A growing body of scientific evidence has linked dairy intake to a reduced type 2 diabetes (T2D) risk. Using an evidence-based approach, we reviewed the most recent and strongest evidence on the relationship between dairy intake and the risk of T2D. Evidence indicates that dairy intake is significantly associated with a reduced T2D risk, and likely in a doseresponse manner. The association between low-fat dairy and T2D risk reduction appears consistent. A beneficial impact is suggested for regular-fat dairy. The role of specific dairy products needs to be clarified. Potential underlying mechanisms include the role of dairy products in obesity and metabolic syndrome, as well as several dairy components, such as calcium, vitamin D, dairy fat, and specifically trans-palmitoleic acid. To conclude, there is strong, consistent, and accumulating evidence that dairy intake reduces the risk of T2D. More research is needed to better understand the role of regular-fat and specific dairy products. Well-designed randomized controlled trials and mechanistic studies are needed to support these findings. Efforts to translate this evidence into clinical practice and public health guidance are needed.

Keywords: dairy products, milk, dairy, diabetes, T2D, metabolic syndrome

\section{INTRODUCTION}

Diabetes is a global epidemic with major health, social, and economic implications. Over the last three decades, the prevalence of diabetes has more than doubled, and more than 371 million people worldwide now have diabetes (1). In 2012, diabetes was the cause of 4.8 million deaths and the estimated health care cost of diabetes was $\$ 471$ billion worldwide (1).

Type 2 diabetes (T2D) accounts for at least $90 \%$ of all diabetes cases and its rates have been increasing at an alarming rate in every country (2). Lifestyle has been identified as playing a major role in the development and prevention of T2D. T2D usually develops during adulthood and is associated with obesity, physical inactivity, and unhealthy diets (2).

A growing body of scientific evidence has linked dairy products to a reduced risk of T2D. Potential underlying mechanisms remain to be fully elucidated but may include a beneficial role of dairy products in obesity and metabolic syndrome (MetS), two important risk factors for T2D, as well as a beneficial role of certain dairy components such as calcium, vitamin D, dairy fat, and specifically trans-palmitoleic acid.

Unfortunately, dairy products remain largely under-consumed by all age groups. An astonishing number of Americans (more than $80 \%$ men and $90 \%$ women) do not meet the minimum dairy requirements of the Dietary Guidelines for Americans (DGA) (3). A large proportion of Canadians also do not meet the minimum daily recommendations established by Health Canada, with an average intake for adults being 1.52 servings, which is well below the $2-4$ recommended daily servings (4). Under-consumption of dairy products has also been reported in many other parts of the world (5-7).

Given the increasing amount of evidence on the beneficial role of dairy products in T2D risk reduction, ensuring optimal dairy intake may represent an additional key strategy against this epidemic. In this review, we aim to examine and synthesize the scientific evidence on the association between dairy products and T2D risk, and identify opportunities for future research. Using a comprehensive evidence-based approach, we reviewed the evidence on the relationship between dairy product intake and the risk of T2D. Online databases were searched until March 2013 for relevant peer-reviewed articles. Priority was given to the most recent and strongest available evidence, with a focus on metaanalyses, randomized controlled trials (RCTs), and prospective cohort studies.

\section{DAIRY AND TYPE 2 DIABETES SYSTEMATIC REVIEWS AND META-ANALYSES}

Three meta-analyses of prospective cohort studies on dairy products and T2D were identified (Table 1). In a meta-analysis by Tong et al. the highest dairy consumption, compared to the lowest category, significantly reduced the risk of T2D by $14 \%$ (combined relative risk (RR) $0.86,95 \%$ confidence interval (CI) 0.79-0.92) (8). A significant inverse association with T2D was also observed for lowfat dairy and yogurt. High-fat dairy and whole (regular-fat) milk were not found to be associated with T2D. A dose-response analysis showed a decrease of $6 \%$ in $\mathrm{T} 2 \mathrm{D}$ risk per each additional daily serving of total dairy (RR $0.94,95 \%$ CI $0.92-0.97$ ). A dose-response 
Table 1 | List of studies reviewed and main findings on dairy products and T2D.

Study Main findings

\section{SYSTEMATIC REVIEWS AND META-ANALYSES}

Kratz et al. (20)

Tong et al. (8)

Elwood et al. (9)

Pittas et al. (10)

2010 Dietary Guidelines for Americans PROSPECTIVE COHORT STUDIES

Grantham et al. (12)

Louie et al. (13)

Struijk et al. (14)

Soedamah-Muthu et al. (15)

Fumeron et al. (16)

Malik et al. (17)

Sluijs et al. (18)

Mozaffarian et al. $(21,22)$
Inverse association between high-fat dairy products and T2D

T2D risk reduction by $14 \%$ for highest diary intake

Significant inverse association for low-fat dairy and yogurt

No association for high-fat dairy and whole milk

Decrease of $6 \%$ in T2D risk per each additional serving of total dairy per day

Decrease of $10 \%$ in T2D risk per each additional serving of low-fat dairy per day

Reduction in diabetes incidence by $4-9 \%$ for each additional daily serving of dairy products

Lower risk of incident T2D for the highest vs. lowest dairy intake (3-5 vs. 1.5 servings per day)

Moderate evidence indicates that dairy products is associated with a reduced risk of T2D

Significant inverse association between the highest tertile of dairy intake and risk of diabetes among men Significant inverse association between low-fat milk and diabetes

No association between total dairy consumption and T2D

No association for reduced/low-fat dairy and regular-fat dairy

Significant reduction by $59 \%$ in risk of MetS with regular-fat dairy

No significant association between total dairy intake and T2D incidence

Beneficial effect of cheese and fermented dairy on glucose regulation indices

No significant association between total dairy consumption or specific types of dairy products and T2D Association of fermented dairy products with reduced risk of overall mortality

Inverse association between total consumption of dairy products and impaired fasting glycemia and T2D Inverse relationship between cheese and incident metabolic syndrome

Significantly reduced risk of T2D by $38 \%$ for highest quintile of dairy consumption during adolescence (two servings per day)

A 43\% reduction in risk of T2D with consistently high intakes of dairy products (from adolescence to adulthood) A $25 \%$ risk reduction for the highest current consumption of dairy products (two servings per 1,000 kcal)

No association between total dairy consumption and T2D

Inverse association between cheese and fermented dairy products and T2D

Significant reduction by $48-62 \%$ in risk of incident T2D with trans-palmitoleic acid

Direct association between dairy fat and trans-palmitoleic acid levels

Inverse association with risk of T2D for whole (regular-fat) dairy across all intake levels relationship between low-fat dairy consumption and T2D was also present, with a $10 \%$ risk reduction per additional serving (RR 0.90, 95\% CI 0.85-0.95).

In their meta-analysis of four prospective cohort studies on diabetes, Elwood et al. demonstrated that milk or dairy consumption was protective against T2D (RR 0.92, 95\% CI 0.86-0.97). Each additional daily serving was significantly associated with a reduction in diabetes incidence by $4-9 \%$ (9). In another metaanalysis with mostly similar cohort studies, the highest vs. lowest dairy intake (3-5 vs. 1.5 servings per day) was associated with a lower risk of incident T2D [odds ratio (OR) 0.86, 95\% CI 0.79 0.93] (10). Furthermore, in the 2010 DGA, the Dietary Guidelines Advisory Committee (DGAC) concluded that moderate evidence indicates that dairy products are associated with a reduced risk of T2D after their systematic review of literature (11).

\section{PROSPECTIVE COHORT STUDIES}

Several prospective cohort studies, published thereafter, have also been conducted to evaluate the potential role of dairy products in T2D prevention (Table 1 ).

The Australian Diabetes Obesity and Lifestyle Study (AusDiab), a population-based, prospective survey with follow-up of 5 years, consisting of 5,582 adults demonstrated a significant inverse association between the highest tertile of dairy intake and risk of diabetes in men, after adjustment for age, sex, energy intake, and various other clinical characteristics (adjusted OR 0.53, 95\% CI 0.29-0.96) (12). Among women, a non-significant inverse association was observed (adjusted OR 0.71, 95\% CI 0.48-1.05). Among subtypes of dairy products (low-fat milk, full-fat milk, yogurt, cheese), only low-fat milk had a significant inverse association with diabetes (adjusted OR 0.65, 95\% CI 0.44-0.94). 
Conversely, in another Australian prospective cohort study, the Blue Mountains Eye Study (BMES), after adjustment for covariates including energy intake, fiber from vegetables, dietary glycemic load, and calcium, total dairy consumption was not found to be associated with T2D (adjusted OR 1.50, 95\% CI 0.474.77 ) (13). No association was also observed for reduced/low-fat dairy (adjusted OR 1.09, 95\% CI 0.57-2.09), or regular-fat dairy (adjusted OR 0.87, 95\% CI 0.48-1.57), and T2D risk. However, compared with subjects in the lowest intake quartile of regular-fat dairy products, those in the highest quartile had a 59\% lower risk of MetS (multivariate adjusted OR 0.41, 95\% CI 0.23-0.71).

A prospective analysis of the Inter99 Study, a Danish population-based lifestyle intervention, assessed the association between specific types of dairy products and T2D incidence. Glucose regulation, an important underlying mechanism in the development of T2D, was also investigated (14). No significant association was found between total dairy intake and T2D incidence (OR 0.95, 95\% CI 0.86-1.06). There was also no association between specific dairy products and T2D, but cheese and fermented dairy appeared to have a beneficial effect on glucose regulation indices. A linear inverse association was demonstrated between cheese and $2 \mathrm{~h}$ plasma glucose $(\beta=-0.048,95 \% \mathrm{CI}$ -0.095 to -0.001 ). Fermented dairy products (cheese, yogurt, and buttermilk) were also inversely associated with fasting plasma glucose and hemoglobin Alc.

The Whitehall II prospective cohort study was a London-based study of the working staff of Civil Service departments (15). Tenyear follow-up of 4,186 participants indicated that total dairy consumption was not significantly associated with T2D [hazard ratio (HR) 1.30, 95\% CI 0.95-1.77]. Neither high-fat dairy, lowfat dairy, total milk, yogurt, cheese nor fermented products were associated with T2D risk. However, fermented dairy products were significantly associated with an inverse risk of overall mortality.

The Data from the Epidemiological Study on Insulin Resistance Syndrome (DESIR) study was a prospective cohort study of French adults, followed for 9 years (16). Adjustment was made for potential confounders including fat intake, and analysis of 3,435 participants demonstrated that total consumption of dairy products, excluding cheese, was inversely associated with incident impaired fasting glycemia and T2D (adjusted OR 0.85, 95\% CI 0.76-0.94). Cheese consumption was not associated with T2D (adjusted OR 0.93, 95\% CI 0.82-1.06), but an inverse relationship was found between cheese and incident MetS (adjusted OR 0.82, 95\% CI 0.71-0.95).

Data from 37,038 women from the Nurses' Health Study II, followed for 7 years, was used to evaluate whether dairy consumption during adolescence was associated with the development of T2D in adulthood (17). After adjustment for risk factors present in adolescence, those in the highest quintile of dairy consumption during adolescence (two servings per day) had a reduced risk of T2D by $38 \%$. Adjusting for risk factors present in adulthood also showed a significant inverse association between adolescent dairy intake and T2D (RR 0.73, 95\% CI 0.54-0.97). A 43\% reduction in risk of T2D was observed for women with consistently high-dairy intakes (from adolescence to adulthood), highlighting the importance of persistence in dairy consumption. A 25\% risk reduction was also observed for the highest current dairy consumption (two servings per $1,000 \mathrm{kcal}$ ), and a 26 and $28 \%$ risk reduction with low- and high-fat dairy, respectively.

In a nested case-cohort analysis within eight countries of the European Prospective Investigation into Cancer and Nutrition (EPIC) Study, consisting of a subcohort of 16,835 participants, total dairy consumption was not associated with T2D (HR 1.01, 95\% CI 0.83-1.34) (18). Both the consumption of cheese and fermented dairy products (cheese, yogurt, and thick fermented milk) were inversely associated with T2D (HR 0.88, 95\% CI 0.76-1.02 and HR 0.88, 95\% CI 0.78-0.99, respectively).

\section{RANDOMIZED CONTROLLED TRIALS}

To our knowledge, no RCTs to date have specifically assessed the association between dairy products and the risk of incident T2D. However, in a randomized crossover trial of 12 months, the consumption of low-fat dairy (four servings per day) was associated with improved insulin resistance, without adversely affecting body weight and lipid status (19).

\section{REGULAR/HIGH-FAT DAIRY}

While the evidence appears to be relatively consistent with respect to a beneficial role of low-fat dairy products in the prevention of $\mathrm{T} 2 \mathrm{D}$, the role of regular/high-fat dairy, as well as dairy fat itself, is less clear. In a recent systematic review of observational studies (20), the majority of studies suggested that high-fat dairy products were inversely associated with T2D, either significantly or non-significantly.

In a prospective cohort analysis of the Cardiovascular Health Study (CHS), trans-palmitoleic acid, a naturally occurring trans fatty acid found in dairy and ruminant fat, was shown to significantly and considerably reduce the risk of incident T2D, with a risk reduction of $62 \%$ (21). Dairy fat content appeared to be directly related to trans-palmitoleic acid levels, and whole-fat but not low-fat dairy, was also inversely associated with the risk of T2D. In another subsequent prospective cohort study, the MultiEthnic Study of Atherosclerosis (MESA), trans-palmitoleic acid was associated with a $48 \%$ lower risk of incident T2D (22). A pooled meta-analysis of these two independent cohorts indicated a $29 \%$ lower incidence of diabetes for each $0.05 \%$ point of higher trans-palmitoleic acid concentrations (22).

\section{SPECIFIC TYPES OF DAIRY PRODUCTS}

The evidence regarding the role of specific types of dairy products such as milk, yogurt, and cheese is limited. Milk has generally been assessed as part of total dairy consumption, and limited evidence exists on milk specifically. It appears that milk consumption may be associated with a reduced risk of T2D, with no association for regular-fat or whole milk and T2D $(8,9,15)$. Limited evidence suggests that yogurt is inversely associated with T2D $(8,12,14)$. Cheese consumption may be associated with a reduced risk of $\mathrm{T} 2 \mathrm{D}$, but this needs to be confirmed as some findings are not statistically significant (14-16, 18). Finally, a protective role of fermented dairy products as a whole (including yogurt, cheese, buttermilk, and fermented milk), is suggested against T2D $(14,18)$. 


\section{POTENTIAL MECHANISMS \\ OBESITY AND WEIGHT}

Including dairy products in weight loss diets has been shown to be favorable by decreasing adiposity, while preserving lean mass. In a meta-analysis of RCTs, high-dairy calorie-restricted diets led to a significantly greater reduction in body weight, waist circumference, and fat mass, while increasing lean mass significantly more than conventional weight loss diets (23). In another meta-analysis, dairy consumption, in the short-term, coupled with calorie-restriction, had a beneficial impact on body fat reduction (24). Two systematic reviews of observational studies also showed that increased dairy consumption may be protective against weight gain (20, 25).

A large prospective analysis of more than 120,000 individuals followed for 12-20 years assessed the role of diet (including dairy) and lifestyle on long-term weight gain and found yogurt to be a factor associated with a beneficial impact on weight (26). This study found no association between high-or-low-fat dairy categories or cheese and weight gain and all liquids, except milk, were associated with weight gain.

The potential mechanisms by which dairy may be protective against weight gain include reduced lipogenesis and increased lipolysis. A high calcium intake may lead to the formation of insoluble soaps by calcium and the prevention of fat absorption (24, $27,28)$. As well, a systematic review of RCTs has confirmed a role of calcium in fat oxidation (29). Whey protein may also play a role in muscle sparing and lipid metabolism $(30,31)$.

\section{METABOLIC SYNDROME}

There is increasing evidence indicating that dairy products may reduce the risk of MetS including a systematic review of observational studies (32). The mechanism appears to be via the effects of different dairy components on specific MetS risk factors, such as blood glucose level and hypertension, amongst others (32).

\section{DAIRY COMPONENTS}

As indicated in Table 2, key dairy components, including calcium, vitamin D, dairy fat, and trans-palmitoleic acid, have been suggested to contribute to the metabolic pathways that may be implicated in the prevention of T2D.

\section{CALCIUM}

Calcium may play a key role in regulating insulin-mediated intracellular processes in insulin-responsive tissues $(10,33,34)$. Insulin secretion is a calcium-dependent process, and changes in $\mathrm{Ca}^{2+}$ flux can adversely affect the secretory function of pancreatic $\beta$-cells (10). The phosphorylation of insulin receptors is also a calciumdependent process $(10,35)$. Changes in intracellular calcium levels modulate adipocyte metabolism, which may suppress insulinmediated lipolysis and promote de novo lipogenesis, thus leading to fat accumulation $(10,36)$.

\section{VITAMIN D}

Circulating active vitamin D may have a direct effect on insulin secretion by binding to vitamin $\mathrm{D}$ receptors in pancreatic $\beta$-cells. Vitamin D also has an indirect effect via the regulation of extracellular calcium for normal calcium flux through cell membranes and adequate $\mathrm{Ca}^{2+}$ pool (37). Vitamin D may enhance insulin receptor expression and insulin responsiveness for glucose transport (38). Moreover, vitamin $\mathrm{D}$ directly activates peroxisome proliferator activator receptor- $\delta$, a transcription factor involved in fatty acid metabolism $(39,40)$.

T2D has also been shown to be associated with systemic inflammation $(41,42)$. Insulin sensitivity and $\beta$-cell survival may be improved through the direct effect of vitamin $\mathrm{D}$ on the generation of cytokines (10). Vitamin D also down-regulates genes encoding pro-inflammatory cytokines involved in insulin resistance (43). The anti-inflammatory mechanism may also be modulated by the regulation of $\mathrm{Ca}^{2+}$ levels (10).

\section{DAIRY FAT AND TRANS-PALMITOLEIC ACID}

The trans isomer of palmitoleic acid (trans-16:1n-7) is a natural source of palmitoleic acid, mainly obtained via the diet from dairy or ruminant fat. Trans-palmitoleic acid may have similar actions to that of endogenous cis-palmitoleic acid $(22,26)$. Up-regulation of adipose-derived cis-palmitoleic acid improves hepatic and peripheral insulin resistance, diminishes metabolic dysregulation, and suppresses hepatic de novo lipogenesis $(20,22,44)$. Higher transpalimitoleic acids levels are also associated with lower triglycerides, fasting insulin, blood pressure, and C-reactive protein $(21,22)$.

Other dairy-derived fatty acids have also been associated with lower T2D risk but findings are less consistent (22). A recent metaanalysis of cohort studies has indicated that dietary saturated fat is not associated with risk of T2D (45).

\section{LIMITATIONS}

Our review of the most recent studies on dairy products and risk of T2D has some limitations. Most of the studies included in this review consisted of prospective cohort studies. The potential for bias in such observational studies is present in that individuals who have a regular consumption of dairy products may also engage in other healthful diet and exercise behavior. In most studies, adjustments were made for potential confounders, yet, the possibility of residual confounding cannot be completely eliminated.

\section{CONCLUSION}

There is a strong and relatively consistent body of accumulating evidence indicating that dairy products may significantly reduce the risk of T2D and likely in a dose-response manner. The protective effect of low-fat dairy products against T2D appears consistent in the literature. Evidence on regular-fat dairy products suggests no association with T2D or a beneficial impact. The role of specific dairy products such as cheese and yogurt appears beneficial.

\section{IMPLICATIONS FOR FUTURE RESEARCH}

More research is needed to better understand the role of regularfat and specific types of dairy products on incidence of T2D and indices of glycemic regulation. Large RCTs are needed to confirm findings on the role of dairy in the management of cardiometabolic risk factors, particularly in at risk population, such as those with prediabetes.

Randomized controlled trials are also warranted to increase our understanding of the role of individual dairy nutrients per se. 
Table 2 | Potential mechanisms of dairy products in preventing type 2 diabetes.

Obesity and weight Favorable effect of dairy products during weight loss through fat mass reduction and preservation/augmentation of lean mass Reduction of lipogenesis and increase in lipolysis by high calcium intake

Prevention of fat absorption via the formation of calcium insoluble soaps

Increased fat oxidation by calcium

Role of whey protein in muscle sparing and lipid metabolism

Metabolic syndrome Reduced risk of MetS via improvement of several cardiometabolic risk factors (e.g., blood lipids, blood pressure, abdominal fat) DAIRY COMPONENTS

Calcium

Regulation of insulin-mediated intracellular processes in insulin-responsive tissues

Secretory function of pancreatic $\beta$-cells

Phosphorylation of insulin receptors

Down-regulation of regulator genes encoding pro-inflammatory cytokines involved in insulin resistance

Vitamin D

Direct effect on insulin secretion by binding to vitamin D receptors in pancreatic $\beta$-cells

Indirect effect via the regulation of extracellular calcium

Protection against systemic inflammation by counteracting cytokine generation

Trans-palmitoleic acid Improvement of hepatic and peripheral insulin resistance, metabolic regulation, and suppression of hepatic de novo lipogenesis Lower levels of triglycerides, fasting insulin, blood pressure, and C-reactive protein

Moreover, mechanistic studies are essential to understand the underlying mechanisms regarding dairy products and specific dairy components in preventing T2D.

\section{IMPLICATIONS FOR PRACTICE}

Given the evidence regarding dairy products in T2D prevention, translation into practice, and clinical and public health guidance is warranted. The value of having an adequate intake of dairy products is to be reinforced especially during weight loss and among those with prediabetes and MetS.

\section{REFERENCES}

1. International Diabetes Federation. IDF Diabetes Atlas Update 2012. 5th ed. (2012). Available from: http:// www.idf.org/

2. Canadian Diabetes Association Clinical Practice Guidelines Expert Committee. Canadian Diabetes Association 2008 clinical practice guidelines for the prevention and management of diabetes in Canada. Can J Diabetes (2008) 32:S1-201.

3. Krebs-Smith SM, Guenther PM, Subar AF, Kirkpatrick SI, Dodd KW. Americans do not meet federal dietary recommendations. J Nutr (2010) 140:1832-8. doi:10.3945/jn. 110.124826

4. Garriguet D. Canadians' eating habits. Health Rep (2007) 18:17-32.

5. Vandevijvere S, De Vriese S, Huybrechts I, Moreau M, Temme E, De Henauw S, et al. The gap between food-based dietary guidelines and usual food consumption in Belgium, 2004. Public Health Nutr (2009) 12:423-31. doi:10. 1017/S1368980008002164

6. Liu AD, Zhang B, Du WW, Wang HJ, $\mathrm{Su}$ C, Zhai FY. [Milk consumption and its changing trend of Chinese adult aged $18-44$ in nine provinces (autonomous region) from 1991 to 2006]. Zhonghua Yu Fang Yi Xue Za Zhi (2011) 45:304-9.

7. Doidge JC, Segal L. Most Australians do not meet recommendations for dairy consumption: findings of a new technique to analyse nutrition surveys. Aust $N Z J P u b$ lic Health (2012) 36:236-40. doi: 10.1111/j.1753-6405

8. Tong X, Dong JY, Wu ZW, Li W, Qin LQ. Dairy consumption and risk of type 2 diabetes mellitus: a meta-analysis of cohort studies. Eur J Clin Nutr (2011) 65:1027-31. doi:

9. Elwood PC, Givens DI, Beswick AD, Fehily AM, Pickering JE, Gallacher J. The survival advantage of milk and dairy consumption: an overview of evidence from cohort studies of vascular diseases, diabetes and cancer. J Am Coll Nutr (2008) 27:723S-34. doi:10.1080/07315724. 2008.10719750

10. Pittas AG, Lau J, Hu FB, DawsonHughes B. The role of vitamin $\mathrm{D}$ and calcium in type 2 diabetes. A systematic review and 10.1038/ejcn.2011.62

Identifying adequate dairy intake as a key strategy in clinical practice guidelines for T2D is also needed. Finally, communicating the importance of meeting dairy recommendations should be an essential part of public health guidance and identifying strategies to increase dairy consumption to optimal levels is of utmost importance.

\section{ACKNOWLEDGMENTS}

The authors would like to thank Isabelle Neiderer and Nathalie Savoie for their review of this manuscript and their input.

meta-analysis. J Clin Endocrinol Metab (2007) 92:2017-29. doi:10. 1210/jc.2007-0298

11. United States Department of Agriculture. Report of the Dietary Guidelines Advisory Committee on the Dietary Guidelines for Americans 2010 to the Secretary of Health and Human Services and the Secretary of Agriculture. Washington, DC (2010).

12. Grantham NM, Magliano DJ, Hodge A, Jowett J, Meikle P, Shaw JE. The association between dairy food intake and the incidence of diabetes in Australia: the Australian Diabetes Obesity and Lifestyle Study (AusDiab). Public Health Nutr (2013) 16:339-45. doi: 10.1017/S1368980012001310

13. Louie JC, Flood VM, Rangan AM, Burlutsky G, Gill TP, Gopinath $\mathrm{B}$, et al. Higher regular fat dairy consumption is associated with lower incidence of metabolic syndrome but not type 2 diabetes. Nutr Metab Cardiovasc Dis (2012). doi:10.1016/ j.numecd.2012.08.004

14. Struijk EA, Heraclides A, Witte DR, Soedamah-Muthu SS, Geleijnse JM, Toft U, et al. Dairy product intake in relation to glucose regulation indices and risk of type 2 diabetes. Nutr Metab Cardiovasc Dis (2012). doi:10.1016/j.numecd.2012. 05.011

15. Soedamah-Muthu SS, Masset G, Verberne L, Geleijnse JM, Brunner EJ. Consumption of dairy products and associations with incident diabetes, CHD and mortality in the Whitehall II study. $\mathrm{Br}$ J Nutr (2012) 109:718-26. doi:10. 1017/S0007114512001845

16. Fumeron F, Lamri A, Abi Khalil C, Jaziri R, Porchay-Balderelli I, Lantieri O, et al. Dairy consumption and the incidence of hyperglycemia and the metabolic syndrome: results from a French prospective study, Data from the Epidemiological Study on the Insulin Resistance Syndrome (DESIR). Diabetes Care (2011) 34:813-7. doi:10.2337/dc101772

17. Malik VS, Sun Q, Van Dam RM, Rimm EB, Willett WC, Rosner B, et al. Adolescent dairy product consumption and risk of type 2 diabetes in middle-aged women. Am J Clin Nutr (2011) 94:854-61. doi: 10.3945/ajcn.110.009621 
18. Sluijs I, Forouhi NG, Beulens JW, van der Schouw YT, Agnoli C, Arriola L, et al. The amount and type of dairy product intake and incident type 2 diabetes: results from the EPIC-InterAct Study. Am J Clin Nutr (2012) 96:382-90. doi: 10.3945/ajcn.111.021907

19. Rideout TC, Marinangeli CP, Martin H, Browne RW, Rempel CB. Consumption of low-fat dairy foods for 6 months improves insulin resistance without adversely affecting lipids or bodyweight in healthy adults: a randomized free-living cross-over study. Nutr J (2013) 12:56. doi:10.1186/1475-2891-1256

20. Kratz M, Baars T, Guyenet S. The relationship between high-fat dairy consumption and obesity, cardiovascular, and metabolic disease. Eur J Nutr (2013) 52:1-24. doi:10.1007/ s00394-012-0418-1

21. Mozaffarian D, Cao H, King IB, Lemaitre RN, Song X, Siscovick DS, et al. Trans-palmitoleic acid, metabolic risk factors, and new-onset diabetes in U.S. adults: a cohort study. Ann Intern Med (2010) 153:790-9. doi:10.7326/0003-4819153-12-201012210-00005

22. Mozaffarian D, De Oliveira Otto MC, Lemaitre RN, Fretts AM, Hotamisligil G, Tsai MY, et al. transPalmitoleic acid, other dairy fat biomarkers, and incident diabetes: the Multi-Ethnic Study of Atherosclerosis (MESA). Am J Clin Nutr (2013) 97(4):854-61. doi:10.3945/ ajcn.112.045468

23. Abargouei AS, Janghorbani M, Salehi-Marzijarani M, Esmaillzadeh A. Effect of dairy consumption on weight and body composition in adults: a systematic review and meta-analysis of randomized controlled clinical trials. Int $J$ Obes (Lond) (2012) 36:1485-93. doi:10. 1038/ijo.2011.269

24. Chen M, Pan A, Malik VS, Hu FB. Effects of dairy intake on body weight and fat: a meta-analysis of randomized controlled trials. Am J Clin Nutr (2012) 96:735-47. doi: 10.3945/ajcn.112.037119

25. Louie JC, Flood VM, Hector DJ, Rangan AM, Gill TP. Dairy consumption and overweight and obesity: a systematic review of prospective cohort studies. Obes Rev (2011) 12:e582-92. doi:10.1111/j. 1467-789X.2011.00881.x

26. Mozaffarian D, Hao T, Rimm EB, Willett WC, Hu FB. Changes in diet and lifestyle and long-term weight gain in women and men. $N$ Engl J Med (2011) 364:2392-404. doi: 10.1056/NEJMoa1014296

27. Zemel MB. The role of dairy foods in weight management. J Am Coll Nutr (2005) 24:537S-46. doi:10. 1080/07315724.2005.10719502

28. Christensen R, Lorenzen JK, Svith CR, Bartels EM, Melanson EL, Saris WH, et al. Effect of calcium from dairy and dietary supplements on faecal fat excretion: a meta-analysis of randomized controlled trials. Obes Rev (2009) 10:475-86. doi: 10.1111/j.1467-789X.2009.00599.x

29. Gonzalez JT, Rumbold PL, Stevenson EJ. Effect of calcium intake on fat oxidation in adults: a metaanalysis of randomized, controlled trials. Obes Rev (2012) 13:84857. doi:10.1111/j.1467-789X.2012. 01013.x

30. Pihlanto-Leppala A, Koskinen P, Piilola K, Tupasela T, Korhonen H. Angiotensin I-converting enzyme inhibitory properties of whey protein digests: concentration and characterization of active peptides. J Dairy Res (2000) 67:53-64. doi: 10.1017/S0022029999003982

31. Pal S, Ellis V, Dhaliwal S. Effects of whey protein isolate on body composition, lipids, insulin and glucose in overweight and obese individuals. Br J Nutr (2010) 104:716-23. doi:10.1017/S0007114510000991

32. Crichton GE, Bryan J, Buckley J, Murphy KJ. Dairy consumption and metabolic syndrome: a systematic review of findings and methodological issues. Obes Rev (2011) 12:e190-201. doi:10.1111/j. 1467-789X.2010.00837.x

33. Ojuka EO. Role of calcium and AMP kinase in the regulation of mitochondrial biogenesis and GLUT4 levels in muscle. Proc Nutr Soc (2004) 63:275-8. doi:10.1079/ PNS2004339
34. Wright DC, Hucker KA, Holloszy JO, Han DH. Ca2+ and AMPK both mediate stimulation of glucose transport by muscle contractions. Diabetes (2004) 53:330-5. doi:10. 2337/diabetes.53.2.330

35. Zemel MB. Nutritional and endocrine modulation of intracellular calcium: implications in obesity, insulin resistance and hypertension. Mol Cell Biochem (1998) 188:129-36. doi: 10.1023/A:1006880708475

36. Zemel MB, Shi H, Greer B, Dirienzo D, Zemel PC. Regulation of adiposity by dietary calcium. FASEB J (2000) 14:1132-8.

37. Bland R, Markovic D, Hills CE, Hughes SV, Chan SL, Squires PE, et al. Expression of 25-hydroxyvitamin D3lalpha-hydroxylase in pancreatic islets. I Steroid Biochem $\mathrm{Mol}$ Biol (2004) 89-90:121-5. doi: 10.1016/j.jsbmb.2004.03.115

38. Maestro B, Campion J, Davila N, Calle C. Stimulation by 1,25 dihydroxyvitamin D3 of insulin receptor expression and insulin responsiveness for glucose transport in U-937 human promonocytic cells. Endocr J (2000) 47:383-91. doi:10.1507/endocrj.47.383

39. Dunlop TW, Vaisanen S, Frank C, Molnar F, Sinkkonen L, Carlberg C. The human peroxisome proliferator-activated receptor delta gene is a primary target of 1alpha,25-dihydroxyvitamin D3 and its nuclear receptor. $J$ Mol Biol (2005) 349:248-60. doi: 10.1016/j.jmb.2005.03.060

40. Luquet S, Gaudel C, Holst D, Lopez-Soriano J, Jehl-Pietri C, Fredenrich A, et al. Roles of PPAR delta in lipid absorption and metabolism: a new target for the treatment of type 2 diabetes. Biochim Biophys Acta (2005) 1740:313-7. doi:10.1016/j.bbadis. 2004.11.011

41. Pradhan AD, Manson JE Rifai N, Buring JE, Ridker PM. C-reactive protein, interleukin 6 , and risk of developing type 2 diabetes mellitus. JAMA (2001) 286:327-34. doi:10.1001/jama.286. 3.327
42. Duncan BB, Schmidt MI, Pankow JS, Ballantyne CM, Couper D, Vigo A, et al. Low-grade systemic inflammation and the development of type 2 diabetes: the atherosclerosis risk in communities study. Diabetes (2003) 52:1799-805. doi:10. 2337/diabetes.52.7.1799

43. Pittas AG, Joseph NA, Greenberg AS. Adipocytokines and insulin resistance. J Clin Endocrinol Metab (2004) 89:447-52. doi:10.1210/jc. 2003-031005

44. Dimopoulos N, Watson M, Sakamoto K, Hundal HS. Differential effects of palmitate and palmitoleate on insulin action and glucose utilization in rat L6 skeletal muscle cells. Biochem J (2006) 399:473-81. doi: 10.1042/BJ20060244

45. Alhazmi A, Stojanovski E, McEvoy M, Garg ML. Macronutrient intakes and development of type 2 diabetes: a systematic review and meta-analysis of cohort studies. $J$ Am Coll Nutr (2012) 31:243-58. doi: 10.1080/07315724.2012.10720425

Conflict of Interest Statement: Maria Kalergis is employed at Dairy Farmers of Canada, a non-profit agri-food organization. Sylvie S. L. Leung Yinko and Roxana Nedelcu have consulted for Dairy Farmers of Canada.

Received: 26 March 2013; paper pending published: 17 April 2013; accepted: 08 July 2013; published online: 23 July 2013. Citation: Kalergis M, Leung Yinko SSL and Nedelcu $R$ (2013) Dairy products and prevention of type 2 diabetes: implications for research and practice. Front. Endocrinol. 4:90. doi: 10.3389/fendo.2013.00090

This article was submitted to Frontiers in Diabetes, a specialty of Frontiers in Endocrinology.

Copyright (c) 2013 Kalergis, Leung Yinko and Nedelcu. This is an open-access article distributed under the terms of the Creative Commons Attribution License, which permits use, distribution and reproduction in other forums, provided the original authors and source are credited and subject to any copyright notices concerning any third-party graphics etc. 